\title{
Composer. Écritures musicales... sur le vif
}

\section{Nicolas Donin}

\section{(2) OpenEdition}

\section{Journals}

Édition électronique

URL : http://journals.openedition.org/genesis/821

DOI : 10.4000/genesis.821

ISSN : 2268-1590

\section{Éditeur :}

Presses universitaires de Paris Sorbonne (PUPS), Société internationale de génétique artistique littéraire et scientifique (SIGALES)

\section{Édition imprimée}

Date de publication : 10 octobre 2010

Pagination : 7-10

ISBN : 978-2-84050-711-6

ISSN : 1167-5101

\section{Référence électronique}

Nicolas Donin, «Composer. Écritures musicales... sur le vif », Genesis [En ligne], 31 | 2010, mis en ligne le 28 mai 2013, consulté le 22 septembre 2020. URL : http://journals.openedition.org/genesis/821 ; DOI : https://doi.org/10.4000/genesis.821

Ce document a été généré automatiquement le 22 septembre 2020.

Tous droits réservés 


\title{
Composer. Écritures musicales... sur le vif
}

\author{
Nicolas Donin
}

Encore un numéro de Genesis sur la musique contemporaine?

2 Un précédent numéro thématique de la revue était en effet consacré, sous la direction du musicologue Peter Szendy, aux «Écritures musicales aujourd'hui » (1993). Coéditée avec l'Ircam (Institut de Recherche et de Coordination Acoustique/Musique), cette quatrième livraison de Genesis réunissait les signatures prestigieuses de certains des compositeurs, théoriciens et musicologues les plus représentatifs de l'activité artistique et pédagogique de l'Institut à cette époque. À travers l'analyse d'œuvres de Boulez, Nono, Stockhausen, mais aussi de compositeurs plus jeunes comme Nunes ou Jarrell, on y scrutait les fruits d'une modernité esthétique héritière non seulement des grands créateurs d'avant-garde du siècle dernier, tels que Debussy ou Schoenberg, mais aussi de toute une tradition littéraire et philosophique qui s'avérait proche des corpus et des référents théoriques mobilisés par la critique génétique : Joyce et le work in progress, le Livre mallarméen ou René Char (Boulez), l’Oulipo (Jarrell), Walter Benjamin (Nono) ou encore Jacques Derrida.

Pendant la petite vingtaine d'années écoulée, la création musicale a foisonné et notre vision du canon moderniste s'est peu à peu transformée. Assurément, les lignes esthétiques ont bougé et il y aurait déjà beaucoup à dire sur les mutations contemporaines des différentes cultures du son au sein desquelles les «écritures musicales aujourd'hui ", devenues plus intimement numériques que jamais, se reconfigurent. Cependant, susciter un second numéro sur la musique contemporaine pour compléter ou pour actualiser le premier n'aurait guère eu de raison d'être dans l'histoire de Genesis. Songeons qu'en matière de musique, la revue n'a encore jamais traité de l'opéra, de l'improvisation ou de la relation musique/texte - sujets, parmi d'autres, essentiels à la conquête d'une théorie génétique générale (par-delà la spécificité du fait littéraire) qui en constitue l'un des fils rouges éditoriaux. Alors, pourquoi cette nouvelle thématique sur la création musicale contemporaine? 
4 Ce qu'avance le présent numéro, c'est en fait bien moins un type d'objet qu'un type de méthode, moins la spécificité de corpus choisis pour eux-mêmes que la communauté d'un type de problématique émergente susceptible d'intéresser tout généticien, musicien ou non : peut-on pratiquer une génétique "sur le vif», saisissant ses objets avant même le moment de leur réception par un public ainsi que par une tradition critique ? Et si oui, comment ? La « musique contemporaine » se trouve avoir été le lieu d'expérimentation des démarches scientifiques que nous soumettons à la curiosité du lecteur; mais nous n'attendons pas de ce dernier un intérêt de principe pour les valeurs propres à tel monde esthétique plutôt qu'à tel autre. Ce qu'il convient d'entendre ici dans le syntagme «musique contemporaine », c'est la contemporanéité comme condition de possibilité d'une proximité chronologique entre la genèse de l'œuvre et son étude génétique. Autrement dit, la création musicale contemporaine nous intéressera ici comme moyen d'accès privilégié à une catégorie particulière de questionnement génétique, distincte de ce à quoi nous a habitués l'étude de processus créateurs temporellement distants. Cela étant, en explorant des compositions musicales dont l'encre est à peine sèche, voire même "en train de se faire " (pour reprendre une expression chère à Pascal Dusapin ${ }^{1}$ ), nous ne ferons que remettre sur l'établi la perspective de recherches «in vivo", plus d'une fois abordée par la critique génétique sans qu'elle ait encore pleinement acquis le rôle de stimulant théorique auquel elle pourrait prétendre, ne serait-ce qu'au titre d'expérience à la limite.

5 Ce dossier naît donc principalement d'une interrogation méthodologique. Mais une autre interrogation vient à sa suite : les travaux qui abordent des processus créateurs résolument "sur le vif» ne nous feraient-ils pas passer d'une face à l'autre de la génétique, c'est-à-dire de l'analyse des documents à celle de l'action et de la cognition ? Car ce qui se présente à nous en premier, dans ce genre d'études, ce sont des actions, des pratiques, des situations; et l'on pourrait se demander si la génétique devient soluble dans l'ethnologie ou dans la sociologie à mesure que ses objets sont proches dans le temps - d'autant que les méthodes introduites dans ce dossier s'inspirent effectivement de telles disciplines "de terrain" (par opposition à des disciplines "d'archive »). Pourtant, on le verra, la génétique in vivo n'est pas plus ou pas moins solidaire d'un projet anthropologique que d'un projet critique et analytique (ici de nature musicale, mais on pourra fréquemment remplacer « musicologie » par « théorie littéraire »). Comme toute spécification du programme d'ensemble de la critique génétique, celle-ci se place sur la crête entre deux versants, ou instances, des processus de genèse : d'une part, les logiques de la production créatrice, dans la particularité de leur médium, dans la singularité de leurs œuvres, dans leur dynamique autonome de transformation de formes symboliques; d'autre part, la cognition du créateur avec ses aspects conscients et inconscients, ses conditions sociohistoriques, ses savoir-faire émergents et ses automatismes aveugles.

6 À l'exploration des faits d'écriture, déjà bien développée dans la critique génétique musicale, devait donc répondre une investigation des manières de composer, donnant toute son importance à l'infinitif ("Composer» et non "Compositions») en mettant l'accent sur l'incertitude du travail créateur², dont les études génétiques sur le vif peuvent espérer documenter l'évolution à chaque étape, non plus seulement sur la base de ses traces écrites mais aussi d'autres formes d'extériorisation - verbales ou non - du processus. 
7 Le beau mot de « composer " sert aussi à marquer un questionnement sur la singularité de la musique dans sa différence avec la littérature. Qu'est-ce qui, des modes d'invention et d'agencement du matériau musical, vaut aussi pour la création littéraire? Quelles opérations, quelles façons de penser sont partagées (et parfois confrontées) par ces deux domaines? Ne perd-on pas davantage qu'on ne gagne à postuler d'emblée l'autonomie irréductible du champ musical, sous le prétexte de sa technicité ostensible ou de son rapport si controversé à la sémantique ? En tout cas, le lexique de la composition est partagé par le poète, le peintre et le musicien - et la volonté d'en théoriser la « méthode » n'aura pas moins préoccupé Poe que Schoenberg. Puissent les textes de ce dossier contribuer, à leur modeste échelle, au vaste travail de clarification conceptuelle qui permettrait d'éclairer réciproquement les logiques compositionnelles des musiciens et celles des écrivains.

8 Le premier de ces textes présente une série de travaux menés à l'Ircam depuis 2003 au sein d'une équipe de recherche en musicologie et sciences humaines. Toute une série d'études a tenté de répondre aux délicates questions théoriques, empiriques et méthodologiques soulevées par le projet, souvent jugé chimérique, d'une génétique du contemporain - une génétique à même de travailler sur des documents d'une fraîcheur et d'une richesse hors du commun, mais devant prendre le risque d'interactions avec le processus de genèse lui-même, sans parler du manque de recul qui menace à tout moment ce type de démarche. Le bilan de ces recherches ne saurait être que provisoire (comme en témoignera assez l'évocation d'une œuvre musicale en cours de composition à l'heure où l'article a été rédigé), mais les avancées obtenues suffisent à proposer un modèle complet et réaliste d'étude génétique in vivo, que ses principes de construction et de réalisation distinguent aussi clairement d'une simple interview d'artiste améliorée que d'une étude de nature strictement anthropologique ou sociologique sur l'activité créatrice.

9 Amanda Bayley présente ensuite une " enquête » sur la genèse d'une œuvre récente de Michael Finnissy. Il s'agit là d'un autre cas de figure: le projet de recherche portait originairement sur les techniques de jeu instrumental du quatuor à cordes contemporain, vues à travers le travail des interprètes du Quatuor Kreutzer sur plusieurs partitions, dont l'une est une œuvre nouvelle du compositeur britannique. Mais une documentation croisée des points de vue complémentaires du compositeur et des membres du quatuor sur la façon de comprendre et de réaliser le texte musical a permis à la chercheuse de repérer un ensemble de traits importants de la genèse de l'œuvre, et ce, en incluant (c'est suffisamment rare en musicologie pour être relevé) les étapes cruciales de l'appropriation de la partition par les interprètes et de la transmission de clés de lecture par le compositeur en vue d'influencer cette interprétation ${ }^{3}$.

10 Le texte de Philippe Leroux a un statut inhabituel, non seulement parce que les compositeurs contemporains prennent rarement la plume (et celui-ci n'échappe pas à cette règle), mais surtout parce qu'il présente, de façon relativement détaillée, le point de vue d'un créateur sur une étude génétique de sa propre production. Contributeur décisif à deux recherches de longue haleine menées à l'Ircam (voir supra) sur la genèse de deux de ses œuvres récentes, Philippe Leroux s'est prêté avec grâce, au fil de plusieurs années, à un dispositif de recueil et d'analyse des données sur son activité créatrice dans lequel sa mémoire et ses savoir-faire musiciens étaient intensivement sollicités, occasionnant un surcroît de travail dans la vie d'un artiste à l'emploi du 
temps déjà particulièrement chargé. Tandis qu'une longue série de publications a marqué les pas gagnés du côté des chercheurs, les effets de l'étude sur son « sujet » (ou plutôt « informateur privilégié ») n'avaient pas encore fait, jusqu'à maintenant, l'objet d'un publié.

11 Suit un entretien avec le compositeur français Georges Aperghis, connu aussi bien pour ses nombreux spectacles de théâtre musical à Avignon et en région parisienne depuis une quarantaine d'années, que pour ses œuvres de musique de chambre ou pour voix soliste (la plus fameuse, Récitations, fut jadis au programme du baccalauréat !), et plus récemment pour une série de spectacles où les dramaturgies théâtrale et musicale s'entremêlent de façon renouvelée à travers l'usage de la vidéo et de traitements électroniques du son. On y lira les féconds paradoxes d'un créateur d'actions et de situations scéniques qui développe pourtant son matériau à partir de combinatoires abstraites, d'un compositeur qui règle les plus petits détails sur le papier mais sait donner toute sa place à l'inattendu surgissant du travail de répétition avec les musiciens. Capable d'appliquer les mêmes disjoncteurs de sens aux notes d'une phrase musicale et aux phonèmes d'une jactation verbale, Aperghis était l'initiateur idéal pour notre questionnement sur ce qui se laisse « composer » à travers les arts des sons et ceux des mots.

Enfin, nous nous réjouissons de publier des documents inédits sur la genèse d'une œuvre fondatrice du répertoire contemporain, et plus particulièrement d'un courant esthétique né en France dans les années soixante-dix et largement internationalisé depuis une vingtaine d'années: la musique spectrale. Malgré la large diffusion de l'œuvre de Gérard Grisey de son vivant et depuis sa mort brutale en 1998, peu d'études génétiques ont été entreprises par les musicologues, et aucune n'a porté sur le cycle Les Espaces acoustiques, l'un des chefs-d'œuvre de la deuxième moitié du siècle dernier. Grisey fut un pionnier de la pensée musicale de notre temps, réalisant, plus de dix ans avant le développement foudroyant de l'informatique musicale à l'échelle industrielle, des va-et-vient d'une grande clairvoyance entre le monde de l'écriture musicale traditionnelle (sur partition destinée à un ensemble d'instrumentistes) et celui de la science acoustique (objectivant le son musical par des moyens de mesure et de représentation basés sur l'analyse de ses propriétés physiques). S'il ne s'agit plus, dans l'exégèse de ces documents inédits, d'une saisie génétique sur le vif, on y trouvera en revanche de belles occasions de méditer sur la singularité du travail des compositeurs de notre époque, tissant patiemment les unes aux autres des sémiotiques aussi différentes que celle de la représentation graphique des fréquences sonores, celles des chiffres et du calcul, et bien sûr celle du solfège.

\section{NOTES}

1. Pascal Dusapin, Une musique en train de se faire, Paris, Seuil, 2009. Ce volume reprend les propos tenus par le compositeur au Collège de France (où il était titulaire de la Chaire de Création artistique en 2007) et les complète par différents textes et entretiens. Pour une discussion de la position de Dusapin, dans sa Leçon inaugurale (p. 101-127), sur l'impossibilité de construire une 
connaissance de la composition, voir Nicolas Donin et Jacques Theureau, "Ateliers en mouvement. Interroger la composition musicale aujourd'hui ", Circuit, Musiques contemporaines, vol. XVIII, $n^{\circ} 1,2008$, p. 5-14.

2. Récemment thématisée par un sociologue, Pierre-Michel Menger, tout au long d'un ouvrage dont un chapitre est consacré aux problématiques de l'esquisse et du non finito (Le Travail créateur. S'accomplir dans l'incertain, Paris, Seuil/Gallimard, 2009).

3. La traduction du texte d'Amanda Bayley a été financée par le projet Musicologie des techniques de composition contemporaines ( $\mathrm{MuTeC})$, soutenu par l'Agence nationale de la recherche (ANR-08-CREA-066). Le présent dossier présente les premiers résultats de ce projet (contributions de Nicolas Donin et de François-Xavier Féron).

\section{INDEX}

Mots-clés : génétique, genèse, création (processus de), musique

\section{AUTEUR}

\section{NICOLAS DONIN}

Nicolas Donin est musicologue à l'Institut de Recherche et de Coordination Acoustique/Musique, où il dirige l'équipe Analyse des pratiques musicales (Ircam-CNRS). Il est l'auteur d'études sur la musique contemporaine, l'histoire des pratiques d'écoute et d'analyse musicale depuis la fin du $\mathrm{XIX}^{\mathrm{e}}$ siècle, et l'analyse musicale d'interprétation, publiées à la fois dans le champ musicologique et dans celui des sciences humaines. Il est aussi coauteur de courts-métrages, de DVD-Roms et de publications en ligne issus de ces recherches.

Nicolas.Donin@ircam.fr 